\begin{tabular}{c} 
Benha Veterinary Medical Journal \\
$\begin{array}{c}\text { Official Journal Issued by } \\
\text { Faculty of } \\
\text { Veterinary Medicine }\end{array}$ \\
\hline
\end{tabular}

Original Paper

\title{
Beneficial anti-inflammatory and anti-arthritic activities of Grape seed proanthocyanidin extract in Freund's Complete Adjuvant-induced Rheumatoid arthritis in rats
}

\author{
Samy A. Hussein ${ }^{1}$, Samir A. Abdelal ${ }^{2}$, Frahat F. Ali $^{3}$ and Mona E. Elbahlol ${ }^{*}$ \\ ${ }^{1}$ Biochemistry Department, Fac. Vet. Med., Benha University, Egypt. \\ ${ }^{2}$ Animal Hygiene, Behavior and Management Department, Fac. Vet. Med., Benha University, Egypt. \\ ${ }^{3}$ Agricultural Biochemistry Department, Faculty of Agriculture, Benha University, Egypt
}

\section{ARTICLE INFO}

\begin{tabular}{l}
\hline Keywords \\
Freund's adjuvant \\
GSPE \\
Histopathology. \\
Inflammatory mediators \\
Rheumatoid arthritis \\
\hline Received $27 / 11 / 2020$ \\
Accepted $18 / 01 / 2021$ \\
Available On-Line \\
01/0/4/2021
\end{tabular}

\begin{abstract}
Rheumatoid arthritis (RA) is a systemic autoimmune inflammatory disorder with massive suffering and permanent joint destruction. The Grape seed proanthocyanidin extract (GSPE) beneficial effects were evaluated in adjuvant-induced arthritis (AIA). RA was induced by 0.1 $\mathrm{mL}$ Freund's complete adjuvant (CFA) that was injected once into the rat's right hind leg footpads. Thirty male rats were equally divided into 5 groups. Group I (Normal control): Rats received no drugs, Group II (CFA- induced arthritis for treatment): Rats injected with $0.1 \mathrm{ml}$ CFA on the $1^{\text {st }}$ day and sacrificed after 29 days of CFA injection, Group III (CFA + GSPE treated): After 14 days of CFA injection, rats treated with GSPE (100 mg kg ${ }^{-1} \mathrm{~b}$ wt./day) for 15 sequential days. Group IV (CFA- induced arthritis for protection): Rats injected with 0.1 $\mathrm{ml} \mathrm{CFA}$ on $15^{\text {th }}$ day of the experiment and sacrificed 15 days later after arthritis induction.

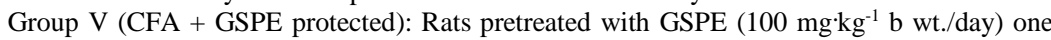
day before CFA injection and 14 days later after arthritis induction. A significant upregulation in IL-1 $\beta$, MMP-3 and LOX-1 gene expressions were observed in the arthritic rats' synovial membrane. However, significant down-regulations in all previous parameters were observed in GSPE treated groups. Also, GSPE significantly activated Nrf2 gene in the liver with marked up-regulation of Lactoferrin (LF) gene in arthritic rats' synovial membrane. Interestingly, improvement of histopathological alterations in synovium and stifle joint capsule of arthritic rats supported the anti-arthritic effects of GSPE. Conclusively, GSPE ameliorates adjuvant induced arthritis via its anti-arthritic and joint protecting properties.
\end{abstract}

\section{INTRODUCTION}

Rheumatoid arthritis (RA) has both articular and systemic effects (McInnes and Schett, 2011), where cytokine imbalance is a pivotal factor in the pathogenesis and progression of this disease. Several experimental models were developed in rats to evaluate the antirheumatic drugs potential benefits (Snekhalatha et al., 2013). The model of complete Freund's adjuvant (CFA)-induced rat arthritis is clinically, immunologically, and pathologically similar to human RA (Kim and Moudgil, 2009). It is marked with bone resorption and proliferation of periosteal bones (Ahmed et al., 2015).Li et al.,(2018), recorded a marked increase in the level of tumor necrosis factor alpha (TNF$\alpha$ ), nuclear factor kappa B (NF-kB), interleukin-1 $\beta$ (IL-1 $\beta$ ) and nuclear factor erythroid 2-related factor $2(\mathrm{Nrf} 2)$ in the arthritic rats. Also, a significant amplification in matrix metalloproteinase-3 (MMP-3) gene expression was detected by Aniss et al., (2020).

Currently, $80 \%$ of the world population utilizes the plantbased medications for successful RA pain relief (Sahoo et al., 2010). GSPE, which belongs to a group of flavonoids, is produced from Vitis vinifera (red grapes) that contains
$95 \%$ of natural proanthocyanidin potent antioxidants (Wren et al., 2002; Wei et al., 2018). GSPE has an anti-arthritic effect in Adjuvant-Induced Arthritis (AIA) model by modifying T cell subsets (Ahmad et al., 2013). Moreover, it activates the Nrf2 pathway (Sun et al., 2016), while inhibiting proinflammatory mediators including TNF- $\alpha$, IL$1 \beta$ (Rajput et al., 2019), transforming growth factor $\beta 1$ (TGF- $\beta 1$ ) (Zhou et al., 2015) and MMP-3 (Khaddam et al., 2014) and the proteolytic enzymes, supporting the GSPE suppressive effects in osteoclasts (Kwak et al., 2020). Accordingly, this study aims to evaluate the anti-arthritic properties and potential protective and therapeutic effect of Grape seed proanthocyanidin extract (GSPE) in Freund's Complete adjuvant-induced rheumatoid arthritis in rats through determination of the mRNA gene expression levels of IL-1 $\beta$, MMP-3, LOX-1, LF and Nrf2 in arthritic rats synovial membrane.

\section{MATERIAL AND METHODS}

\subsection{Experimental animals:}

Thirty white albino male rats, 4-5 weeks old with average weight of $150-200 \mathrm{~g}$ were used in this study. Rats were

\footnotetext{
* Corresponding author: Mona Elmowafy Elbahlol; E-mail: monsci2015@yahoo.com
} 
handled according to the suggested National Ethical Guidelines for the care of Laboratory Animals, as the Animal Ethics Committee of Faculty of Veterinary Medicin, Benha University, Egypt. The animals were left for 2 weeks for acclimatization before the beginning of the experiment.

\subsection{Chemicals and antioxidant agent:}

The antioxidant and chemicals used in the present study were

\subsubsection{Freund's complete adjuvant}

Freund's complete adjuvant (CFA) was bought from SIGMA Chemical Company (USA) containing $10 \mathrm{mg} / \mathrm{ml}$ of heat-killed M. tuberculosis $(10 \mathrm{mg} / \mathrm{ml})$. It was injected once subcutaneously ( $0.1 \mathrm{~mL}$ CFA) into the right hind leg footpads of rats (Nasuti et al., 2019).

\subsubsection{Grape Seed Proanthocyanidin Extract (GSPE):}

Grape Seed Proanthocyanidin Extract was purchased from (Al Debeiky Pharma Company for Pharmaceutical industries, Al Obour, Cairo, Egypt). GSPE was dissolved in $100 \%$ Dimethyl sulfoxide (DMSO) and diluted to the appropriate concentration by sterilized saline solution then administered orally in a dose of (100 mg kg-1 b wt./day) (Ahmad et al., 2013).

\subsection{Experimental design}

The rats were randomly divided into five major equal groups of six rats each placed in individual cages and graded as follows:

Group I: Normal control group: Rats received no drugs, provided with ordinary diet for 29 successive days. They served as an untreated control group for all experimental groups.

Group II: CFA- induced arthritis for treatment: AIA was induced in rats on the first day by a single subcutaneous injection of $0.1 \mathrm{ml}$ of CFA in right hind leg footpad of rats, which were sacrificed 29 days later after CFA injection.

Group III: (CFA + GSPE treated): Rats received GSPE (100 mgk k ${ }^{-1} \mathrm{~b}$ wt./day, orally) for 15 successive days, after 14 days of CFA injection and were then sacrificed.

Group IV: (CFA- induced arthritis for protection): Rats injected with $0.1 \mathrm{ml} \mathrm{CFA}$ in right hind leg footpad on the $15^{\text {th }}$ day of the experiment and were sacrificed 15 days later after arthritis induction.

Group V: (CFA + GSPE protected): Rats were pretreated with GSPE (100 mg kg-1 b wt./day) one day prior to CFA injection, on the $14^{\text {th }}$ day, followed by 15 days later after arthritis induction, then were sacrificed at $\left(30^{\text {th }}\right.$ day).

\subsection{Sampling (Livers and stifle joints)}

At the end of the trial, rats were anesthetized and sacrificed by cervical decapitation according to Animal Ethics Committees, stifle joints and livers tissues were collected once from all rat groups on $30^{\text {th }}$ day of the experiment.

The livers and stifle joints of all rat groups were removed and cleaned by rinsing with cold saline, put in Eppendorf tubes and immediately kept in liquid nitrogen and stored at $-80^{\circ} \mathrm{C}$ till RNA extraction for the molecular analysis of nuclear factor erythroid 2-related factor 2 (Nrf2) gene expression in liver tissue and interleukin-1 $\beta$ (IL-1 $\beta$ ), matrix metalloproteinase 3 (MMP-3), lectin-like oxidized lowdensity lipoprotein receptor-1 (LOX-1) and lactoferrin (LF) in synovial membrane of stifle joints by reverse transcription polymerase chain reaction (RT-PCR).
Moreover, rats stifle joint were taken and immediately fixed in $10 \%$ neutral formalin solution, the washed samples were dehydrated by using graded ascending concentrations of ethyl alcohol starting with 50\% and ending with absolute alcohol, then blocked in hard paraffin and cut into sections of approximately 5 microns thick. Paraffin was removed from the sections that were subsequently stained with Harris hematoxylin and eosin for histopathology examination according to the technique described by (Bancroft and Gamble technique, 2008).

\subsection{Molecular analysis}

The mRNA expressions content of Nrf2 in rat livers and IL$1 \beta$, MMP-3, LOX-1 and LF in the synovial membrane of stifle joint of arthritic rats were determined using real time quantitative polymerase chain reaction (real-time qPCR). Samples were processed simultaneously, as previously described (Bush et al., 2001). $\beta$-actin was used as the load control. Total RNA was isolated using High Kit for pure RNA isolation (Thermo Scientific, Fermentas, \#K0731). The produced cDNAs from the reverse transcribed template RNAs using Revert Aid ${ }^{\mathrm{TM}} \mathrm{H}$ Minus Reverse transcriptase kit (\#EP0451, Thermo Scientific, Fermentas, USA) were amplified on Faststart Universal SYBR Green Master (Roche, GER). The target genes were normalized with $\beta$ Actin by the $2^{-\Delta \Delta C t}$ method (Livak and Schmittgen, 2001).

\begin{tabular}{lcc}
\multicolumn{3}{l}{ Table 1 Forward and reverse primers sequence for real time PCR } \\
\hline Gene & Forward primer & Reverse primer \\
\hline IL-1 $\beta$ & CACCTCTCAAGCAGAGCACAG & GGGTTCCATGGTGAAGTCAAC \\
MMP-3 & AGC AAG GAC CTC GTT TTC ATT & GTC AAT CCC TGG AAA GTC TTC A \\
LF & ACAAGGCGAGACTCGTTCAG & CGATCGCTACGACGTACTCC \\
Nrf2 & CACATCCAGACAGACACCAGT & CTACAAATGGGAATGTCTCTGC \\
LOX-1 & CATGGGCCCTTTAACTGGGA & GAGAGCCGTTCTCCCATAGC \\
$\beta$-actin & AAGTCCCTCACCCTCCCAAAAG & AAGCAATGCTGTCACCTTCCC \\
\hline
\end{tabular}

\subsection{Statistical Analysis}

Results were expressed as mean \pm SE using SPSS (18.0 software, 2011). Using one-way ANOVA followed by Duncan's test, data were analyzed. Values were statistically significant at $p<0.05$

\section{RESULTS}

The data presented in table (2) revealed a significant upregulation $(\mathrm{P} \leq 0.05)$ in IL-1 $\beta$, MMP-3, and LOX -1 gene expression levels, and down-regulation in LF gene expression level in the synovium of the adjuvant injected rats (G2 and G4) compared with the normal controls (G1). The gene expression levels of IL-1 $\beta$, MMP-3, and LOX-1 were significantly down-regulated following administration of GSPE either before (G5) or after (G3) induction of arthritis, with lowest expression in the protected group (G5). Moreover, administration of GSPE elevated LF gene expression level in both (G3) and (G5), with highest expression within the protected cluster (G5), in comparison to the arthritic control groups (G2 and G4).

The obtained results conferred in table (3) showed that Nrf2 gene expression level within the arthritic rat livers was significantly elevated following administration of GSPE either before (G5) or after (G3) induction of RA, with highest expression in the protected cluster (G5), as compared with the arthritic control groups (G2 and G4). 
Table 2 Effect of GSPE administration on synovial membrane IL-1 $\beta$, MMP-3, LOX-1 and LF gene expression levels in Freund's Complete Adjuvant-induced Rheumatoid arthritis in rats.

\begin{tabular}{|c|c|c|c|c|c|c|c|c|}
\hline \multirow[t]{2}{*}{ Animal Groups } & \multicolumn{2}{|l|}{ IL-1 $\beta$} & \multicolumn{2}{|l|}{ MMP-3 } & \multicolumn{2}{|l|}{ LOX-1 } & \multicolumn{2}{|l|}{ LF } \\
\hline & Fold change mean & SEM & Fold change mean & SEM & Fold change mean & SEM & Fold change mean & SEM \\
\hline Group I: Normal control group & $1.00^{\mathrm{f}}$ & 0 & $1.00^{\mathrm{f}}$ & 0 & $1.00^{\mathrm{f}}$ & 0 & $1.00^{\mathrm{a}}$ & 0 \\
\hline Group III: CFA + GSPE treated & $2.38^{\mathrm{d}}$ & 0.13 & $3.51^{\mathrm{d}}$ & 0.17 & $3.20^{\mathrm{d}}$ & 0.19 & $0.61^{\mathrm{c}}$ & 0.04 \\
\hline Group IV: CFA- induced arthritis for protection & $6.02^{\mathrm{a}}$ & 0.31 & $10.48^{\mathrm{a}}$ & 0.48 & $6.06^{\mathrm{a}}$ & 0.37 & $0.08^{\mathrm{e}}$ & 0.01 \\
\hline Group V: CFA + GSPE protected & $1.91^{\mathrm{e}}$ & 0.13 & $2.38^{\mathrm{e}}$ & 0.13 & $2.20^{\mathrm{e}}$ & 0.11 & $1.04^{\mathrm{a}}$ & 0.07 \\
\hline
\end{tabular}

Table 3 Effect of GSPE administration on liver tissue Nrf2 gene expression level in Freund's Complete Adjuvant-induced Rheumatoid arthritis in rats.

\begin{tabular}{lll}
\hline Animal Groups & Nuclear factor erythroid 2-related factor 2 (Nrf2) \\
& Fold change mean & \pm SEM \\
\hline Group I: Normal control group & $1.00^{\mathrm{f}}$ & 0 \\
Group II: CFA- induced arthritis for treatment & $1.72^{\mathrm{e}}$ & 0.1 \\
Group III: CFA + GSPE treated & $4.23^{\mathrm{c}}$ \\
Group IV: CFA- induced arthritis for protection & $1.95^{\mathrm{e}}$ \\
Group V: CFA + GSPE protected & $8.06^{\mathrm{a}}$ & 0.24 \\
\hline Data are presented as (Mean \pm SEM). SEM = Standard error mean. Mean values with different superscript letters in the same column are significantly different at (P $\leq 0.05)$.
\end{tabular}

Microscopical examination of the stifle joint tissues obtained from normal control rats (Group I) revealed normal histological appearance of synovium and stifle joint capsule with an absence of inflammation; this joint preserved also normal architecture with intact articular cartilage and a well-defined joint space as shown in Figure (1).

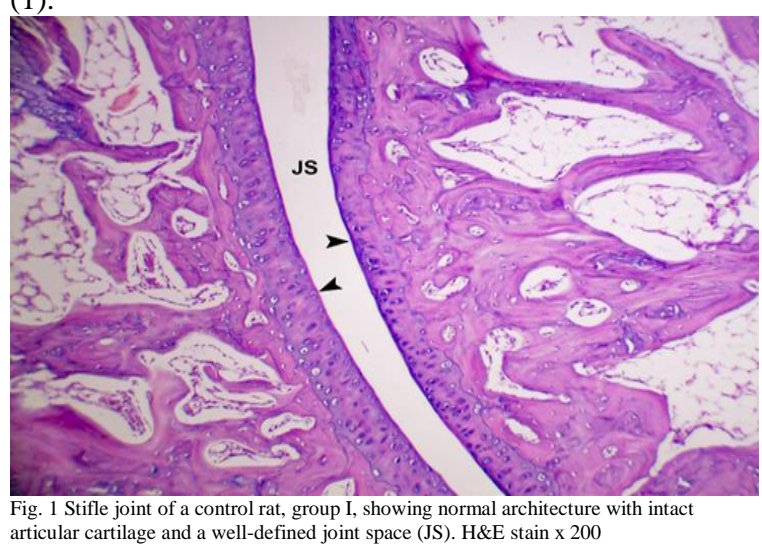

The examined synovium of the affected stifle joint obtained from CFA treated rats (Group II) revealed various histopathological alterations including pannus formation; the synovium was diffusely and markedly thickened and formed numerous wide papillary villar projections. Occasionally, hyperplastic synovial villi contain large multinucleated cells. The proliferated synovial cells and infiltrating pannus markedly eroded the subchondral bone and articular cartilage as highlighted in Figure (2a). Macrophages, lymphocytes and few plasma cells mainly infiltrated the fibrous joint capsule as seen in Figure (2b).

Meanwhile, microscopical examination of stifle joint obtained from the treated group with GSPE (Group III), revealed moderate improvement in its histology. The synovium was mildly thickened without any signs of inflammation. The formation of synovial edema and few papillary villar projections was also observed Figure (3). Macrophages and lymphocytes mainly infiltrated the fibrous joint capsule.
The affected stifle joint obtained from CFA treated rats in (Group IV), showed similar histopathological alterations as Group II, with mild erosion of the articular cartilage at the joint margins by the infiltrating pannus as illustrated in Figure (4).
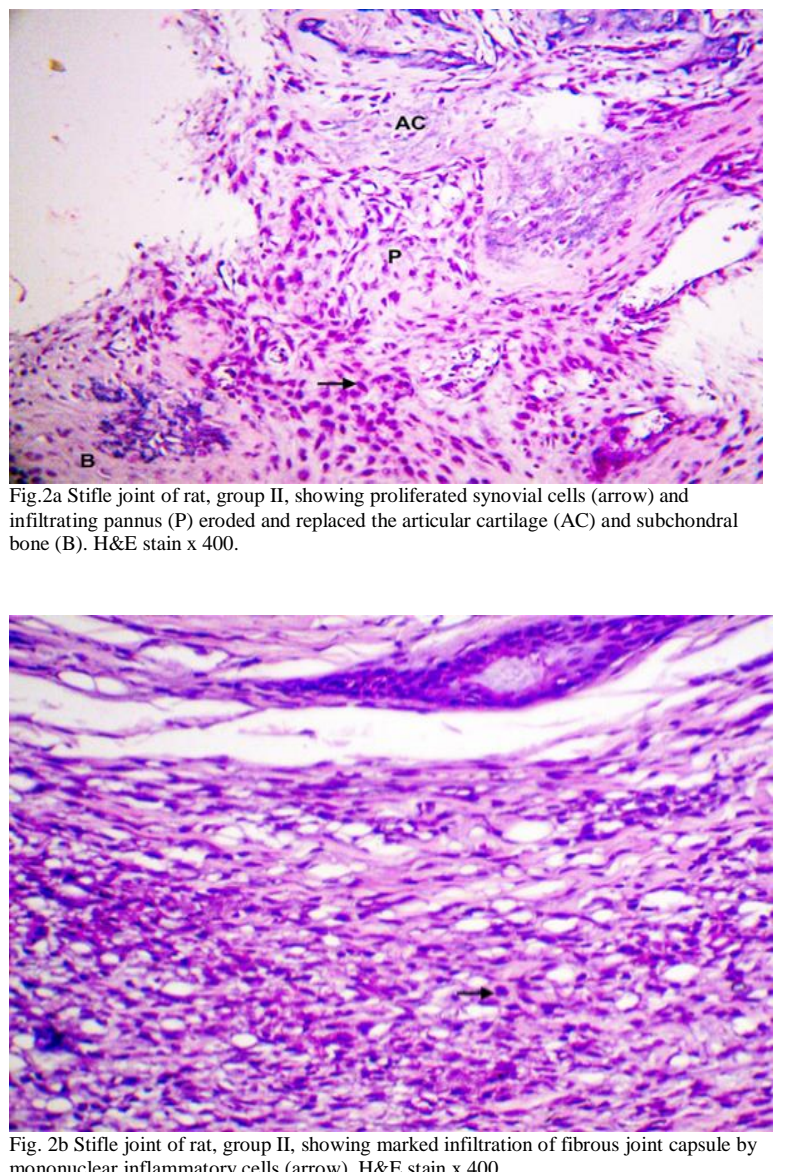

Fig. 2 S Stifle joint of rat, group II, showing marked infiltrat
mononuclear inflammatory cells (arrow). H\&E stain x 400 . 


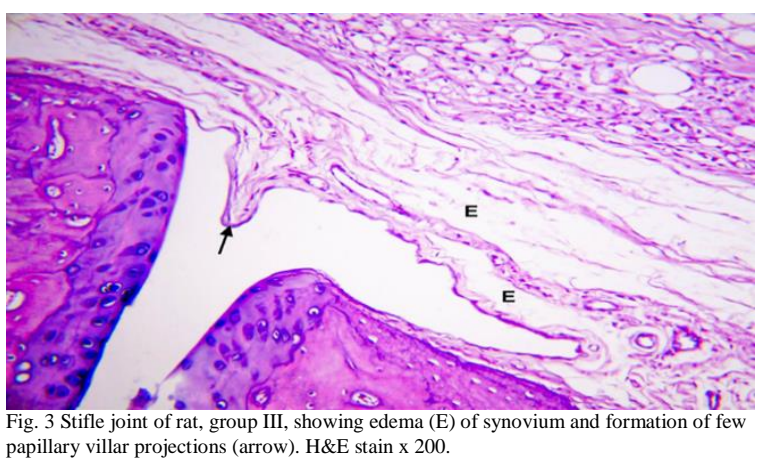

Histopathological examination of the stifle joint obtained from the protected group with GSPE (Group V) revealed edema of the synovium and formation of a few papillary villar projections lined by hypertrophied and hyperplastic synovial cells without signs of inflammation as demonstrated in Figure (5). Macrophages and lymphocytes mainly infiltrated the fibrous joint capsule.

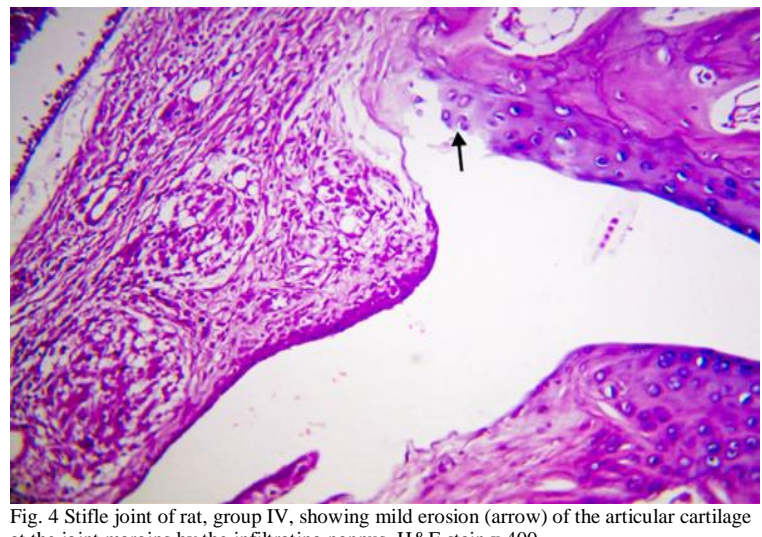

at the joint margins by the infiltrating pannus. H\&E stain $x 400$.

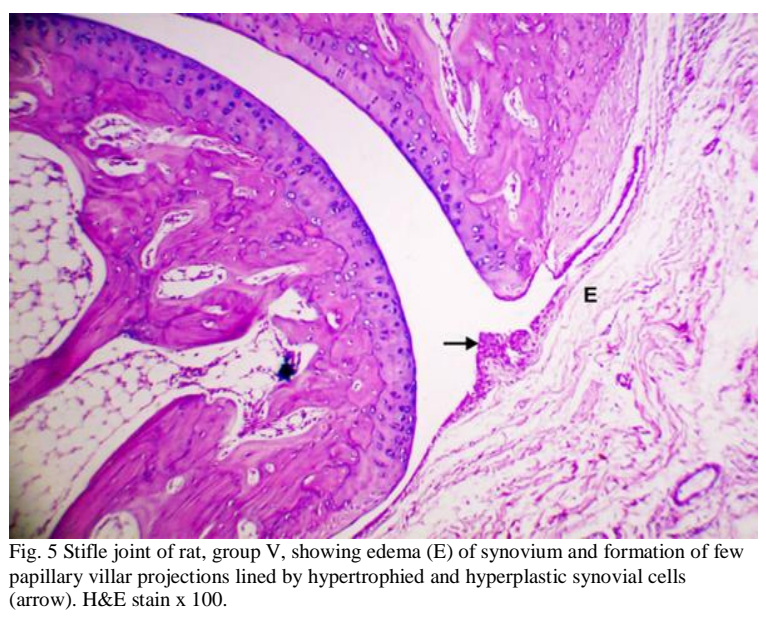

\section{DISCUSSION}

The antigenic CFA induce the immune system by stimulating the production of cellular mediators and certain immunoglobulins (Mythilypriya et al., 2008). Generally, the immune-stimulatory efficiency of CFA is not comparable by any adjuvant, as it is considered the gold standard adjuvant (Fontes et al., 2017), with its simple administration. The CFA- induced arthritis severity causing permanent joint malformations like ankylosis. Therefore, a
CFA rats model was used in this study to evaluate the GSPE potency in treating and preventing RA.

The presented data revealed a significant up-regulation $(\mathrm{P} \leq$ 0.05 ) in IL-1 $\beta$, MMP-3, and LOX - 1 gene expression levels, and down-regulation in LF gene expression level in the synovial membrane of the adjuvant injected rats (G2 and G4) as compared with the normal controls (G1). Similarly, Zeb et al., (2017) showed that injection of CFA subcutaneously in rat feet significantly induced the gene expression of TNF- $\alpha$ (317-fold) and IL-1 $\beta$ (40.66- fold) in paw tissues. Moreover, a significant upregulation $(\mathrm{p}<$ 0.05 ) in MMP-3 gene expression and in serum levels of TNF- $\alpha$ and IL- $1 \beta$ versus the normal control group were demonstrated in adult male rats injected with 0.1 CFA (Aniss et al., 2020).

TNF- $\alpha$ and IL- $1 \beta$ enhance fibroblast proliferation, activate neutrophils, they additionally can increase macrophage infiltration into the inflamed sites with excessive autoantibody production (Kaneko et al., 2001) and increase the other cytokines' expression. IL- $1 \beta$ is 100 times more powerful than TNF $\alpha$ in agitating cartilage degradation in vivo. So IL-1 $\beta$ was thought to be a pivotal link between cartilage destruction and synovium inflammation. While the external osteoid layer removal was mediated by MMP3 , allowing osteoclasts to be attached to the underlying bone that enables bone resorption (Cunnane et al., 2001). Moreover, MMP-3 induces the proteolytic activation of the pro-matrix metalloproteinase-9 (pro-MMP-9) that promotes recruitment of $\mathrm{T}$ cells, neutrophils, monocytes, and osteoclasts (Jones et al., 2008) and membrane-bound Vascular Endothelial Growth Factor (VEGF) releasing, which leads to angiogenesis and disease progression (Szekanecz and Koch, 2009).

Furthermore, Ox-LDL (oxidized low-density lipoprotein) and its receptor LOX-1 which were detected within the RA synovium and in its chondrocytes (Nakagawa et al., 2002) were resulting in increasing the mRNA production of monocyte chemoattractant protein-1 (MCP-1) (Akagi et al., 2009) and VEGF. Levels of MMPs including metalloproteinase-1 (MMP-1) as well as MMP-3 were elevated upon stimulation of fibroblast synovial cells with ox-LDL (Kakinuma et al., 2004), which occurred primarily via the NF- $\kappa \mathrm{B}$ signaling pathway. Toll-like receptor 4 (TLR4) of macrophages elevated the LOX-1 expression and the uptake of oxLDL Hossain et al., (2015), thus TGF$\beta$, interleukin 6 (IL-6), and MCP-1 were released (Van Tits et al., 2011). These data supported the obtained results, which clarified that the LOX-1 gene expression level was significantly increased in the synovial membrane of AIA rats (G2 and G4).

Meanwhile, in the existing study gene expression levels of IL-1 $\beta$, MMP-3, and LOX-1 in the synovial membrane of AIA rats were significantly down-regulated following administration of GSPE either before (G5) or after (G3) induction of arthritis, with the lowest expression in the protected group (G5). These results are harmonized with Ahmad et al., (2014) who clarified the anti-inflammatory activity of GSPE since its administration increased the mRNA expressions of interleukin 10 (IL-10) and TGF- $\beta$, while reducing the IL- $1 \beta$ and TNF- $\alpha$ levels. Also, Khaddam et al., (2014) revealed that GSPE inhibited MMP-3. Additionally, Shao et al., (2020) mentioned that treatment with proanthocyanidins, significantly downregulated the mRNA expression level of LOX-1. Therefore, GSPE markedly suppressed inflammation and consequently 
arthritis progression in the rat model, as confirmed by histopathological findings that showed GSPE suppressed soft tissue and synovial membrane inflammations, so considerably reduced bone erosion and joint degradation.

The current study revealed a significant $(\mathrm{P} \leq 0.05)$ downregulation of LF in synovial membrane of CFA injected rats (G2 and G4) compared to the normal control group (G1). The major component within the secondary neutrophil granules lactoferrin (LF), exhibits antibacterial, antiviral, anti-fungal properties, inhibits several proinflammatory cytokines production, including IL-1 $\beta$, IL-6, interleukin 8(IL-8) and TNF- $\alpha$. It also transfers to the nucleus, so prevents NF- $\mathrm{KB}$ activation (Haversen et al., 2002). It can also promote anti-inflammatory mediators production including IL-10, interleukin4 (IL-4) (Zimecki et al., 2005).

Moreover, the administration of GSPE elevated LF gene expression level in both (G3) and (G5), with the highest expression within the protected cluster (G5), in comparison to the arthritic control groups (G2 and G4). Also, microscopic examination of the stifle joint obtained from the treated and protected rat groups with GSPE, revealed moderate improvement in its histology. The synovium was mildly thickened and formed a few papillary villar projections lined by hypertrophied and hyperplastic synovial cells without any signs of inflammation.

The transcription factor Nrf2 promotes the transcription of various anti-oxidant enzymes upon binding to the antioxidant response element (ARE) on the DNA, thereby reducing the pro-inflammatory pathways (Jung and Kwak, 2010). If any oxidative stress condition is present, such as ROS and/or inflammation, the Nrf2 / HO-1 antioxidant pathway can be activated (Kobayashi et al., 2013). A slightly increased HO-1 and Nrf2 expression levels are detected in patients with RA and in CIA mice (Wruck et al., 2011). The reduced Nrf2 level is a factor that can lead to RA, along the increased levels of TNF- $\alpha$, IL- $1 \beta$ and IL17 , as Nrf2 prevents the production of these proinflammatory cytokines. The relation between NF$\kappa \mathrm{B} / \mathrm{IL}-1 \beta$ and Nrf2 was suggested to be a promoter for the oxidative injury and joint destruction in animals (VomhofDeKrey and Picklo, 2012). Nrf2 also assessed to inhibit the inflammation by keeping the NF- $\mathrm{BB}$ signaling via the upregulation of HO-1 (Bellezza et al., 2012).

In the present study, CFA- treated rats showed significant up-regulation in Nrf2 gene in the arthritic rats' liver. Similar data were recorded by $\mathrm{Wu}$ et al., (2016) who reported that injecting rats with CFA induced RA, significantly elevated Nrf2 expression in rats' synovial tissues ( $p$ < 0.001) compared with the controls, however, its level markedly increased in the inflamed joints of treated groups from RA when compared with the control AIApositive group. In the current study, the hepatic Nrf2 gene expression level in arthritic rats was significantly elevated following administration of GSPE either before (G5) or after (G3) induction of arthritis, when compared with the arthritic control groups (G2 and G4). Similarly, Liu et al., (2017) showed that the protein and the gene expression of Nrf2 in the rat livers were markedly up-regulated as a result of GSPE treatment. Also, Nazima et al., (2020) revealed that GSPE treatment (100 mg kg-1 b wt. for 4 weeks) notably normalized the renal expression of Nrf2/Keap1 (Kelch-like erythroid cell-derived protein with CNC homology $[\mathrm{ECH}]$-associated protein 1) and its downstream regulatory proteins. They suggested that GSP ameliorates oxidative stress through the activation of Nrf2 pathway.
Overall, the results of this study demonstrated that GSPE not only inhibits the key inflammatory factors in RA but also stimulates crucial anti-inflammatory mediators, which in turn support further evaluation for these antioxidant compounds as a treatment for AIA and RA. Thus, the GSPE had therapeutic effects against RA by inhibiting IL$1 \beta$, MMP-3 and LOX-1 gene expression levels in the AIA rats' synovial membrane, while it up-regulated their LF gene expression levels. Moreover, it activates the gene expression level of $\mathrm{Nrf} 2$ in arthritic rats' livers.

\section{CONCULOSIONS}

It could be concluded that, GSPE as a natural compound may provide a safe promising alternative or complimentary anti-rheumatic substance that ameliorates RA in rat joints. Also, GSPE has a strong anti-inflammatory agent that ameliorates RA of rats joint through modulation of LF and inhibiting IL-1 $\beta$, MMP-3, and LOX-1 signaling pathways. So, these results confirm the strong anti-arthritic and joint protective effect of GSPE in arthritis.

\section{ACKNOWLEDGEMENT}

A special thanks for Prof. Dr. Shawky A. A. Moustafa, Head of Pathology Department, Faculty of Vet. Medicine, Benha University for his great assistance in performing histopathological examination for the stifle joint tissue samples, taking the histopathological photos and recording their reports.

\section{REFERENCES}

1. Ahmad, S.F., Zoheir, K.M., Abdel-Hamied, H.E., Ashour, A.E., Bakheet, S.A., Attia, S.M., Abd-Allah, A.R., 2013. Grape seed proanthocyanidin extract has potent anti-arthritic effects on collagen-induced arthritis by modifying the T cell balance. International Immunopharmacology 17(1), 79-87.

2. Ahmad, S.F., Zoheir, K.M., Abdel-Hamied, H.E., Attia, S.M., Bakheet, S.A., Ashour, A.E., Abd-Allah, A.R., 2014. Grape seed proanthocyanidin extract protects against carrageenan-induced lung inflammation in mice through reduction of pro-inflammatory markers and chemokine expressions. Inflammation, 37(2), 500-511.

3. Ahmed, O.M., EL-Abd, S.F., El Mahdi, E.A., Abdou, E.A. 2015. Curcumin ameliorative efficacy on type 1 diabetes mellitus coexisted with rheumatoid arthritis in Wistar rats. Merit Res J Med Med Sci 3(7), 256-70.

4. Akagi, M., Ueda, A., Teramura, T., Kanata, S., Sawamura, T., Hamanishi, C. 2009. Oxidized LDL binding to LOX-1 enhances MCP-1 expression in cultured human articular chondrocytes. Osteoarthritis and cartilage, 17(2), 271-275.

5. Aniss, N.N.D., Zaazaa, A.M., Saleh, M.R.A. 2020. Antiarthritic Effects of Platelets Rich Plasma and Hyaluronic Acid on Adjuvant-induced Arthritis in Rats. International Journal of Pharmacology 16(1), 33-46.

6. Bancroft, J.D. and Gamble, M. 2008. Theory and Practice of Histological Techniques. 6th Edition, Churchill Livingstone, Elsevier, China.

7. Bellezza, I., Tucci, A., Galli, F., Grottelli, S., Mierla, A. L., Pilolli, F., Minelli, A., 2012. Inhibition of NF- $\kappa B$ nuclear translocation via HO-1 activation underlies $\alpha$-tocopheryl succinate toxicity. The Journal of nutritional biochemistry 23(12), 1583-1591. 
8. Bush, K.A., Walker, J.S., Lee, C.S., Kirkham, B.W., 2001. Cytokine expression and synovial pathology in the initiation and spontaneous resolution phases of adjuvant arthritis: Interleukin-17 expression is upregulated in early disease. Clinical \& Experimental Immunology 123(3), 487495.

9. Cunnane, G., FitzGerald, O., Beeton, C., Cawston, T. E., Bresnihan, B. 2001. Early joint erosions and serum levels of matrix metalloproteinase 1 , matrix metalloproteinase 3 , and tissue inhibitor of metalloproteinases 1 in rheumatoid arthritis. Arthritis \& Rheumatism 44(10), 2263-2274.

10. Fontes, J.A., Barin, J.G., Talor, M.V., Stickel, N., Schaub, J., Rose, N.R., Čiháková, D., 2017. Complete Freund's adjuvant induces experimental autoimmune myocarditis by enhancing IL-6 production during initiation of the immune response. Immunity, inflammation and disease 5(2), 163176.

11. Haversen, L., Ohlsson, B. G., Hahn-Zoric, M., Hanson, L. $\AA$ A., Mattsby-Baltzer, I. 2002. Lactoferrin down-regulates the LPS-induced cytokine production in monocytic cells via NF-кB. Cellular immunology 220(2), 83-95.

12. Hossain, E., Ota, A., Karnan, S., Takahashi, M., Mannan, S.B., Konishi, H., Hosokawa, Y. 2015. Lipopolysaccharide augments the uptake of oxidized LDL by up-regulating lectin-like oxidized LDL receptor-1 in macrophages. Molecular and cellular biochemistry 400(1-2), $29-40$.

13. Jones, G.C., Riley, G.P., Buttle, D.J., 2008. The role of proteases in pathologies of the synovial joint. The international journal of biochemistry \& cell biology, 40(67), 1199-1218.

14. Jung, K.A., \& Kwak, M.K., 2010. The Nrf2 system as a potential target for the development of indirect antioxidants. Molecules 15(10), 7266-7291.

15. Kakinuma, T., Yasuda, T., Nakagawa, T., Hiramitsu, T., Akiyoshi, M., Akagi, M., Sawamura, T., Nakamura, T., 2004. Lectin-like oxidized low-density lipoprotein receptor 1 mediates matrix metalloproteinase 3 synthesis enhanced by oxidized low-density lipoprotein in rheumatoid arthritis cartilage. Arthritis \& Rheumatism 50(11), 3495-3503.

16. Kaneko, M., Tomita, T., Nakase, T., Ohsawa, Y., Seki, H., Takeuchi, E., Takano, H., Shi, K., Takahi, K., Kominami, E., et al., 2001. Expression of proteinases and inflammatory cytokines in subchondral bone regions in the destructive joint of rheumatoid arthritis. Rheumatology 40(3), 247-255.

17. Khaddam, M., Salmon, B., Le Denmat, D., Tjaderhane, L., Menashi, S., Chaussain, C., Rochefort, G.Y., Boukpessi, T., 2014. Grape seed extracts inhibit dentin matrix degradation by MMP-3. Frontiers in physiology 5, 425 .

18. Kim, E.Y., \& Moudgil, K.D., 2009. The determinants of susceptibility/resistance to adjuvant arthritis in rats. Arthritis Research \& Therapy 11(4), 239.

19. Kobayashi, E., Suzuki, T., Yamamoto, M., 2013. Roles nrf2 plays in myeloid cells and related disorders. Oxidative medicine and cellular longevity 529219.

20. Kwak, S.C., Cheon, Y.H., Lee, C.H., Jun, H.Y., Yoon, K.H., Lee, M.S., Kim, J.Y., 2020. Grape Seed Proanthocyanidin Extract Prevents Bone Loss via Regulation of Osteoclast Differentiation, Apoptosis,

Proliferation. Nutrients 12(10), 3164.

21. Li, Y., Kakkar, R., Wang, J., 2018. In vivo and in vitro approach to anti-arthritic and anti-inflammatory effect of crocetin by alteration of nuclear factor-E2-related factor 2/hem oxygenase (HO)-1 and NF- $\mathrm{kB}$ expression. Frontiers in Pharmacology, 9, 1341 .

22. Liu, W., Zhao, S., Wang, J., Shi, J., Sun, Y., Wang, W., Guang Ning, G., Hong, J., Liu, R., 2017. Grape seed proanthocyanidin extract ameliorates inflammation and adiposity by modulating gut microbiota in high-fat diet mice. Molecular nutrition \& food research 61(9), 1601082.

23. Livak, K.J. and Schmittgen, T.D., 2001. Analysis of relative gene expression data using real-time quantitative PCR and the 2- ${ }^{\triangle \Delta C T}$ Method. Methods 25(4), 402-408.

24. McInnes, I.B., \& Schett, G., 2011. The pathogenesis of rheumatoid arthritis. New England Journal of Medicine 365(23): 2205-2219.

25. Mythilypriya, R., Shanthi, P., Sachdanandam, P., 2008. Salubrious effect of Kalpaamruthaa, a modified indigenous preparation in adjuvant-induced arthritis in rats- a biochemical interactions 173(2), 148-158.

26. Nakagawa, T., Akagi, M., Hoshikawa, H., Chen, M., Yasuda, T., Mukai, S., Ohsawa, K., Masaki, T., Nakamura, T., Sawamura, T., 2002. Lectin-like oxidized low-density lipoprotein receptor 1 mediates leukocyte infiltration and articular cartilage destruction in rat zymosan-induced arthritis. Arthritis \& Rheumatism 46(9), 2486-2494

27. Nasuti, C., Fedeli, D., Bordoni, L., Piangerelli, M., Servili, M., Selvaggini, R., Gabbianelli, R., 2019. AntiInflammatory, anti-arthritic and anti-nociceptive activities of Nigella sativa oil in a rat model of arthritis. Antioxidants $8(9), 342$

28. Nazima, B., Manoharan, V., Miltonprabu, S., 2020 Retraction: Grape seed proanthocyanidins ameliorates cadmium-induced renal injury and oxidative stress in experimental rats through the up-regulation of nuclear related factor 2 and antioxidant responsive elements. Biochem Cell Biol 89(2), 307.

29. Rajput, S.A., Sun, L., Zhang, N.Y., Khalil, M. M., Ling, Z., Chong, L., Wang, S., Rajput, I.R., Bloch, D.M., Khan, F.A., et al., 2019. Grape seed proanthocyanidin extract alleviates aflatoxinB1-induced immunotoxicity and oxidative stress via modulation of $\mathrm{NF}-\mathrm{\kappa B}$ and $\mathrm{Nrf2}$ signaling pathways in broilers. Toxins 11(1), 23.

30. Sahoo N., Manchikanti P., Dey S., 2010. Herbal drugs: standards and regulation. Fitoterapia 81, 462-471.

31. Shao, D., Di, Y., Lian, Z., Zhu, B., Xu, X., Guo, D., Huang, Q., Jiang, C., Kong, J., Shi, J., 2020. Grape seed proanthocyanidins suppressed macrophage foam cell formation by miRNA-9 via targeting ACAT1 in THP-1 cells. Food \& Function, 11(2), 1258-1269.

32. Snekhalatha, U., Anburajan, M., Venkatraman, B., Menaka, M., 2013. Evaluation of complete Freund's adjuvantinduced arthritis in a Wistar rat model. Zeitschrift für Rheumatologie 72(4), 375-382.

33. Sun, Y., Xiu, C., Liu, W., Tao, Y., Wang, J., Qu, Y.I., 2016. Grape seed proanthocyanidin extract protects the retina against early diabetic injury by activating the Nrf2 pathway. Experimental and therapeutic medicine 11(4), 1253-1258.

34. Szekanecz, Z., \& Koch, A.E., 2009. Angiogenesis and its targeting in rheumatoid arthritis. Vascular pharmacology 51(1), 1-7.

35. Van Tits, L.J.H., Stienstra, R., Van Lent, P.L., Netea, M.G., Joosten, L.A.B., Stalenhoef, A.F.H., 2011. Oxidized LDL enhances pro-inflammatory responses of alternatively activated M2 macrophages: a crucial role for Krüppel-like factor 2. Atherosclerosis 214(2), 345-349.

36. Vomhof-DeKrey, E.E., \& Picklo Sr, M.J., 2012. The Nrf2antioxidant response element pathway: a target for regulating energy metabolism. The Journal of nutritional biochemistry, 23(10), 1201-1206.

37. Wei, M., Guo, F., Rui, D., Wang, H., Feng, G., Li, S., Song, G., 2018. Alleviation of arsenic-induced pulmonary oxidative damage by GSPE as shown during in vivo and in vitro experiments. Biological trace element research 183(1), 80-91. 
38. Wren, A.F., Cleary, M., Frantz, C., Melton, S., Norris, L., 2002. 90-day oral toxicity study of a grape seed extract (IH636) in rats. J Agric Food Chem 50, 2180.

39. Wruck, C.J., Fragoulis, A., Gurzynski, A., Brandenburg, L.O., Kan, Y.W., Chan, K., Joachim Hassenpfl ug, J., Freitag-Wolf, S., Varoga, D., Lippross, S., et al., 2011. Role of oxidative stress in rheumatoid arthritis: insights from the Nrf2-knockout mice. Annals of the rheumatic diseases 70(5), 844-850.

40. Wu, W.J., Jia, W.W., Liu, X.H., Pan, L.L., Zhang, Q.Y., Yang, D., Shen, X., Lium L., Zhu, Y.Z., 2016. Spropargyl-cysteine attenuates inflammatory response in rheumatoid arthritis by modulating the Nrf2-ARE signaling pathway. Redox biology 10, 157-167.

41. Zeb, A., Qureshi, O.S., Yu, C.H., Akram, M., Kim, H.S. Kim, M.S., Kang, J., Majid, A., Chang, S., Bae, O., Kim, J.
K. 2017. Enhanced anti-rheumatic activity of methotrexateentrapped ultradeformable liposomal gel in adjuvantinduced arthritis rat model. International journal of pharmaceutics 525(1), 92-100.

42. Zhou, D.Y., Fang, S.R., Zou, C.F., Zhang, Q., Gu, W., 2015. Proanthocyanidin from grape seed extract inhibits airway inflammation and remodeling in a murine model of chronic asthma. Natural Product Communications 10(2), 1934578X1501000210.

43. Zimecki, M., Artym, J., Chodaczek, G., Kocieba, M., Kruzel, M. 2005. Effects of lactoferrin on the immune response modified by the immobilization stress. Pharmacol. Rep 57, 811-817. 